\title{
Specific absorption rate ratio patterns of cylindrical ultrasound transducers for breast tumors
}

\author{
Win-Li Lin \\ Center for Biomedical Engineering, National Taiwan University, Taipei, Taiwan \\ Jia-Yush Yen \\ Department of Mechanical Engineering, National Taiwan University, Taipei, Taiwan \\ Yung-Yaw Chen \\ Department of Electrical Engineering, National Taiwan University, Taipei, Taiwan \\ Kung-Shan Cheng and Ming-Jium Shieh ${ }^{\text {a) }}$ \\ Center for Biomedical Engineering, National Taiwan University, Taipei, Taiwan
}

(Received 6 May 1997; accepted for publication 31 March 1998)

\begin{abstract}
The purpose of this paper is to examine the optimal driving frequency and to configure the ultrasound energy deposition schema for a various size and location of breast tissues when a portion or the entire cylindrical ultrasound transducer is employed for breast hyperthermia treatments. This work employs a computer simulation program based on an ideal ultrasound power deposition from a cylindrical transducer. The ultrasound power within the breast is assumed to be exponentially attenuated according to the penetration depth of the ultrasound beam and a uniform absorption for the entire breast is also assumed. The distribution of the specific absorption rate (SAR) ratio is employed to determine the heating pattern of a set of given parameters. The control parameters considered are the ultrasound frequency in the breast tissue, the active portion of cylindrical transducer, and the shifting distance between the central axes of the breast and the transducer. The effect of the breast size on the SAR ratio is also considered. Simulation results demonstrate that the breast size, the ultrasound frequency in breast tissue, the shifting distance, and the active portion of the cylindrical transducer are the potential parameters for influencing the distribution of the SAR ratio. High frequencies should be used for the superficial heating treatments and the active portion of the transducer can be changed to obtain a region with an appropriate SAR ratio to cover the treatment region. Low frequencies are used for deep heating treatments and the region of the high SAR ratio can be moved by shifting the transducer and its pattern is varied with the transducer's active portion. The distribution of the SAR ratio indicates the domain of treatable tumor size and tumor depth for a given set of parameters (driving frequency, shifting distance and active portion of the transducer, as well as breast diameter). Findings of this study can be used to know whether or not the tumor is treatable as well as to select the optimal driving frequency and the appropriate active portion of the cylindrical transducer for a treatment, and hopefully to design an appropriate cylindrical ultrasound heating system for breast tumors. (C) 1998 American Association of Physicists in Medicine. [S0094-2405(98)01406-0]
\end{abstract}

Key words: cylindrical ultrasound transducer, breast tumors, treatable domain, optimal frequency, shifting distance, active portion

\section{INTRODUCTION}

Mitsumori et al. ${ }^{1}$ employed an ultrasound hyperthermia system with an improved planar transducer and the frequency scanning technique to minimize the hot spots and to provide improved specific absorption rate (SAR) distribution. The phase I and II clinical trials revealed that 17 (55\%) exhibited CR and $11(35 \%)$ PR for the 30 evaluable breast tumors. A more advanced hyperthermia treatment system with a cylindrical transducer array applicator mounted on a threedimensional translation table has been developed and assessed by Lu and Hansen et al. ${ }^{2,3}$ The applicator consists of 384 ultrasound transducers, each $15 \times 15 \mathrm{~mm}$, mounted on the inner surface of a $25-\mathrm{cm}$-diam cylinder. The optimized transducer frequencies are $2.5 \mathrm{MHz}$ for the low frequency and $4.0-4.5$ for the high frequency. The system is designed to heat individual quadrants or the complete breast to a uniform temperature distribution. The simulation results demonstrate that a combination of low and high frequencies can deliver an appropriate power deposition to achieve and maintain a uniform temperature distribution for a target area from a quadrant to a whole breast. The agreement between theoretical calculations and experimental results based on nonperfused tissue mimicking breast phantoms is excellent. Some other ultrasound hyperthermia systems can penetrate soft tissues to produce deep heating ${ }^{4-7}$ or can be employed for superficial heating. ${ }^{8-10}$

This work employs a relatively simple model for cylindrical ultrasound transducers to examine the relationship be- 
tween the parameters and the distribution of the specific absorption rate (SAR) ratio in the breast. Here the SAR ratio is the ratio of the SAR for a point within the breast to that for a specific point on the breast surface. The ultrasound beam in the breast is attenuated according to the exponential law and a uniform absorption is assumed for the entire breast. The parameters, which might influence the distribution of the SAR ratio and are investigated, are the breast size, ultrasound frequency in the breast tissue, combination of ultrasound frequencies, the shifting distance, and the active portion of the transducer. The simulation results demonstrate that (1) high frequencies should be used for the superficial heating treatments, and the variation of the active portion of the transducer can be used for obtaining a region with an appropriate SAR ratio to cover the treatment region; (2) low frequencies are appropriate for deep heating treatments and the region of high SAR ratio can be moved by shifting the transducer and its pattern is varied with the active portion of the transducer; (3) the combination of low and high frequencies can be used to obtain a portion or the entire breast with a more uniform SAR ratio. The results presented here are valuable for designing an appropriate ultrasound heating system for breast tumors and implementing the treatment planning.

\section{METHODS AND MATERIALS}

The deposition of ultrasound energy within the breast is modeled as a cylindrical transducer with uniform power emitted from the active portion of the transducer face as shown in Fig. 1(a). The breast is taken as a bell shape with different diameters at different depths and surrounded by this transducer. The central axis of the transducer is shifted a distance of $d$ meters away from that of breast along the $x$ axis, shown in Fig. 1(b). The polar coordinates $(r, \theta)$, with respect to the $o^{\prime}-x^{\prime}-y^{\prime}$ system, are used to describe the power deposition within the breast. The ultrasonic beam is straight and convergent propagating to the central axis of the transducer and then divergently spreads. The deposition of ultrasonic intensity at each cylindrical shell with respect to the transducer central axis was simplified as a uniform distribution to obtain a relatively simple but realistic approximation and avoid overly complicated calculations. Moreover the ultrasound power within the breast is assumed to be exponentially attenuated according to the penetration depth of the ultrasound beam. A uniform absorption of ultrasound for the entire breast is also assumed.

\section{A. One-dimensional power deposition}

As the central axis of the transducer is coincident with that of the breast $(d=0)$, the ultrasound power deposition within the breast is one dimensional, symmetrical to the central axis. The control parameter for the power deposition is the ultrasound frequency or attenuation $(\mu)$ in the tissue. (The acoustic attenuation in tissue changes as a function of frequency and tissue properties. For most tissue this change can be approximated as directly proportional to frequency, and hence the following equations and figures are labeled
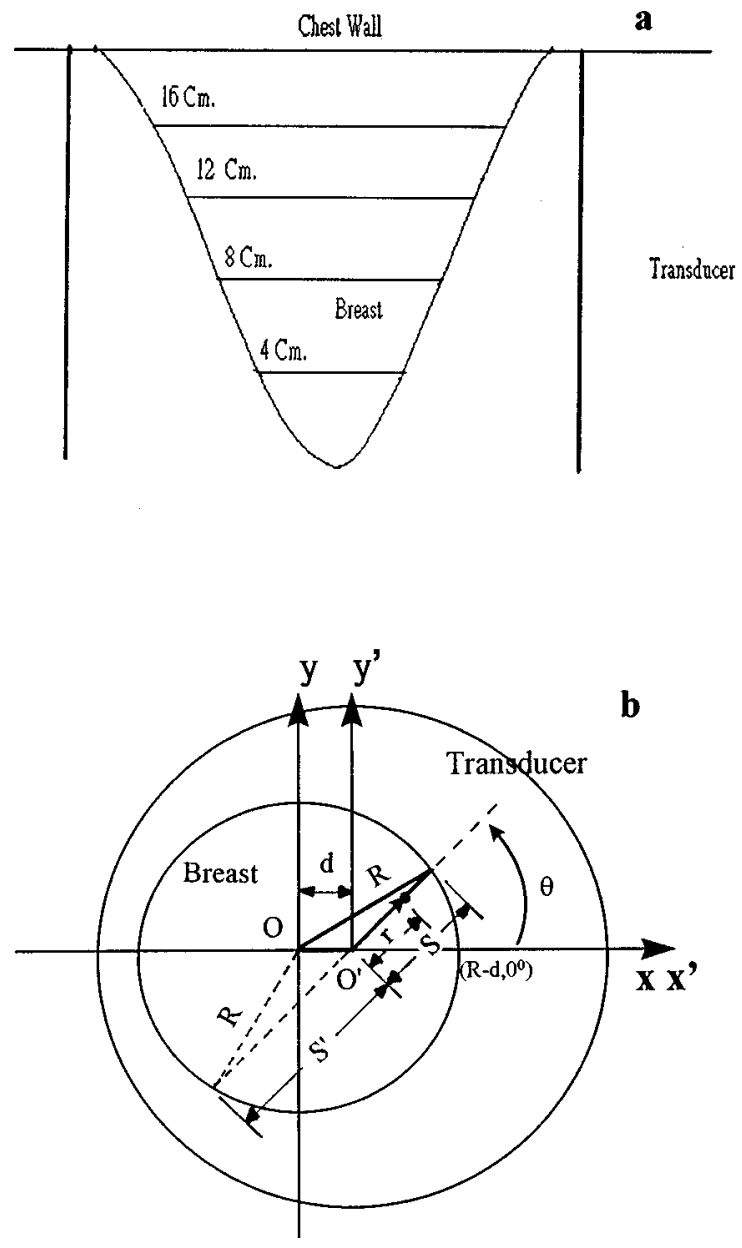

FIG. 1. (a) Schematic diagram of the geometry studied for the distribution of the SAR ratio within a breast while a cylindrical ultrasound transducer is used for the hyperthermia treatment. The breast is assumed as a bell shape with a diameter of $16 \mathrm{~cm}$ in the bottom, $4 \mathrm{~cm}$ close to the apex, and 8 and $12 \mathrm{~cm}$ for the middle. The breast is placed in a cylindrical ultrasound transducer with an emitting power $Q$ for unit length. (b) The relative position between the cylindrical transducer and the breast. The central axis of the transducer is shifted a distance of $d$ away from that of breast along the $x$ axis. The polar coordinates $(r, \theta)$ is used and the SAR value at the point $\left(R-d, 0^{\circ}\right)$ is taken as the reference for the distribution of the SAR ratio.

with attenuation. Once the optimal attenuation in the breast tissue is known, we can obtain the optimal driving frequency.) The ultrasound power propagating through a unit length of a cylindrical shell with radius $r$ meter within the breast is

$$
Q(\mu, R ; r)=Q \cdot\left[e^{-2 \mu(R-r)}+e^{-2 \mu(R+r)}\right],
$$

where $\mu$ is the attenuation of ultrasound in breast tissue; $R$ denotes the breast's radius under investigation; $Q$ represents the ultrasound power emitted from a unit length of the cylindrical transducer; $Q(\mu, R ; r)$ denotes the ultrasound power propagating through a unit length of a cylindrical shell with radius $r$ meter, the surface's area is equal to $A(r)=2 \pi r$.

The ultrasound intensity at the point located on the cylindrical shell with radius $r$ meter within the breast is

$$
I(\mu, R ; r)=\frac{Q \cdot\left[e^{-2 \mu(R-r)}+e^{-2 \mu(R+r)}\right]}{2 \pi r} .
$$


The ultrasound intensity on the breast surface is

$$
I(\mu, R ; R)=\frac{Q \cdot\left[1+e^{-4 \mu R}\right]}{2 \pi R} .
$$

Assuming that the ultrasound intensities are insufficiently large to cause wave distortion and that the attenuation and absorption for breast tissue are equal (all of the attenuated energy is absorbed in the heating field); ${ }^{11}$ then the SAR in homogeneous and uniformly attenuative media is proportional to the ultrasound intensity. Hence, we can obtain the ratio of the SAR on the cylindrical shell with radius $r$ meter within the breast to that on the breast surface as

$$
\begin{aligned}
& \operatorname{SR}(\mu, R ; r ; R) \\
& =\frac{\operatorname{SAR}(\mu, R ; r)}{\operatorname{SAR}(\mu, R ; R)} \\
& =\frac{I(\mu, R ; r)}{I(\mu, R ; R)} \\
& =\mathrm{GG}(r ; R) \cdot \frac{e^{-2 \mu(R-r)}+e^{-2 \mu(R+r)}}{1+e^{-4 \mu R}}, \\
& \mathrm{GG}(r ; R)=\frac{A(R)}{A(r)}=\frac{R}{r},
\end{aligned}
$$

where $\mathrm{GG}(r ; R)$ and $\mathrm{SR}(\mu, R ; r ; R)$ denote the geometrical gain and the ratio of SAR on the cylindrical shell with radius $r$ meter within the breast to that on the breast surface, respectively.

\section{B. Two-dimensional power deposition}

As the central axis of the transducer is shifted a distance (d) away from that of breast, the ultrasound power deposition within the breast becomes two dimensional. The control parameters for the power deposition are the ultrasound frequency [or ultrasound attenuation in breast tissue $(\mu)$ ] and the shifting distance $(d)$. The polar coordinate $(r, \theta)$, with respect to the $o^{\prime}-x^{\prime}-y^{\prime}$ system, is then used to describe the power deposition.

The power deposition at the point $(r, \theta)$ within the breast is contributed by the ultrasound beams from both the front and back directions as shown in Fig. 1(b). $S(R, d ; \theta)$ and $S^{\prime}(R, d ; \theta)$, the penetrating distances of the ultrasound beams from the front and back directions, respectively, to the central axis of the transducer with an angle $\theta$, can be obtained by solving

$$
S^{2}+2 d S \cos \theta+d^{2}-R^{2}=0
$$

and

$$
S^{\prime 2}-2 d S^{\prime} \cos \theta+d^{2}-R^{2}=0 .
$$

The ultrasound intensity at the point $(r, \theta)$ within the breast is

$$
I(\mu, R, d ; r, \theta)=\frac{Q \cdot\left[e^{-2 \mu(S-r)}+e^{-2 \mu\left(S^{\prime}+r\right)}\right]}{2 \pi r}
$$

and that on the breast surface at the point $\left(R-d, 0^{\circ}\right)$ is

$$
I\left(\mu, R, d ; R-d, 0^{\circ}\right)=\frac{Q \cdot\left(1+e^{-4 \mu R}\right)}{2 \pi(R-d)} .
$$

The ratio of the SAR for a point $(r, \theta)$ within the breast to that for the point $\left(R-d, 0^{\circ}\right)$ on the breast surface is

$$
\begin{aligned}
& \operatorname{SR}\left(\mu, R, d ; r, \theta ; R-d, 0^{\circ}\right)= \frac{\operatorname{SAR}(\mu, R, d ; r, \theta)}{\operatorname{SAR}\left(\mu, R, d ; R-d, 0^{\circ}\right)} \\
&= \frac{I(\mu, R, d ; r, \theta)}{I\left(\mu, R, d ; R-d, 0^{\circ}\right)} \\
&= G G\left(r, \theta ; R-d, 0^{\circ}\right) \\
& \cdot \frac{e^{-2 \mu(S-r)}+e^{-2 \mu\left(S^{\prime}+r\right)}}{1+e^{-4 \mu R}}, \\
& G G\left(r, \theta ; R-d, 0^{\circ}\right)=\frac{R-d}{r},
\end{aligned}
$$

where $\mathrm{GG}\left(r, \theta ; R-d, 0^{\circ}\right)$ and $\mathrm{SR}\left(\mu, R, d ; r, \theta ; R-d, 0^{\circ}\right)$ denote the geometrical gain and the ratio of SAR at the location $(r, \theta)$ within the breast to that on the breast surface at $(R$ $\left.-d, 0^{\circ}\right)$, respectively.

For a cylindrical transducer with an active portion $(\varphi$ $<360^{\circ}$ ), symmetrical to the $x^{\prime}$ axis, the control parameters for the ultrasound power deposition are $\mu, d$, and $\varphi$. For a point $(r, \theta)$ within the breast, we consider the ultrasound beams from the front and/or back directions.

For the point $(r, \theta)$ located within the region $-\varphi / 2 \leqslant \theta$ $\leqslant \varphi / 2$ of the breast, the power intensity caused by the ultrasound beam from the front direction is

$$
I(\mu, R, d, \varphi ; r, \theta)=\frac{Q \cdot e^{-2 \mu(S-r)}}{\varphi / 180 \cdot \pi r} .
$$

For the point $(r, \theta)$ located within the region $180^{\circ}-\varphi / 2$ $\leqslant \theta \leqslant 180^{\circ}+\varphi / 2$ of the breast, the power intensity caused by the ultrasound beam from the back direction is

$$
I(\mu, R, d, \varphi ; r, \theta)=\frac{Q \cdot e^{-2 \mu\left(S^{\prime}+r\right)}}{\varphi / 180 \cdot \pi r} .
$$

The ultrasound intensity at $\left(R-d, 0^{\circ}\right)$ on the breast surface is

$$
I\left(\mu, R, d, \varphi ; R-d, 0^{\circ}\right)=\frac{Q}{\varphi / 180 \cdot \pi(R-d)} .
$$

The ratio of the SAR for the point $(r, \theta)$ within the breast to that at $\left(R-d, 0^{\circ}\right)$ on the breast surface is as follows.

(a) For the point $(r, \theta)$ of the breast facing the transducer's active portion, $-\varphi / 2 \leqslant \theta \leqslant \varphi / 2$ :

$\operatorname{SR}\left(\mu, R, d, \varphi ; r, \theta ; R-d, 0^{\circ}\right)$

$$
\begin{aligned}
& =\frac{I(\mu, R, d, \varphi ; r, \theta)}{I\left(\mu, R, d, \varphi ; R-d, 0^{\circ}\right)} \\
& =\mathrm{GG}\left(r, \theta ; R-d, 0^{\circ}\right) \cdot e^{-2 \mu(S-r)},
\end{aligned}
$$

$\mathrm{GG}\left(r, \theta ; R-d, 0^{\circ}\right)=\frac{R-d}{r}$.

(b) For the point $(r, \theta)$ of the breast backing to the trans- 

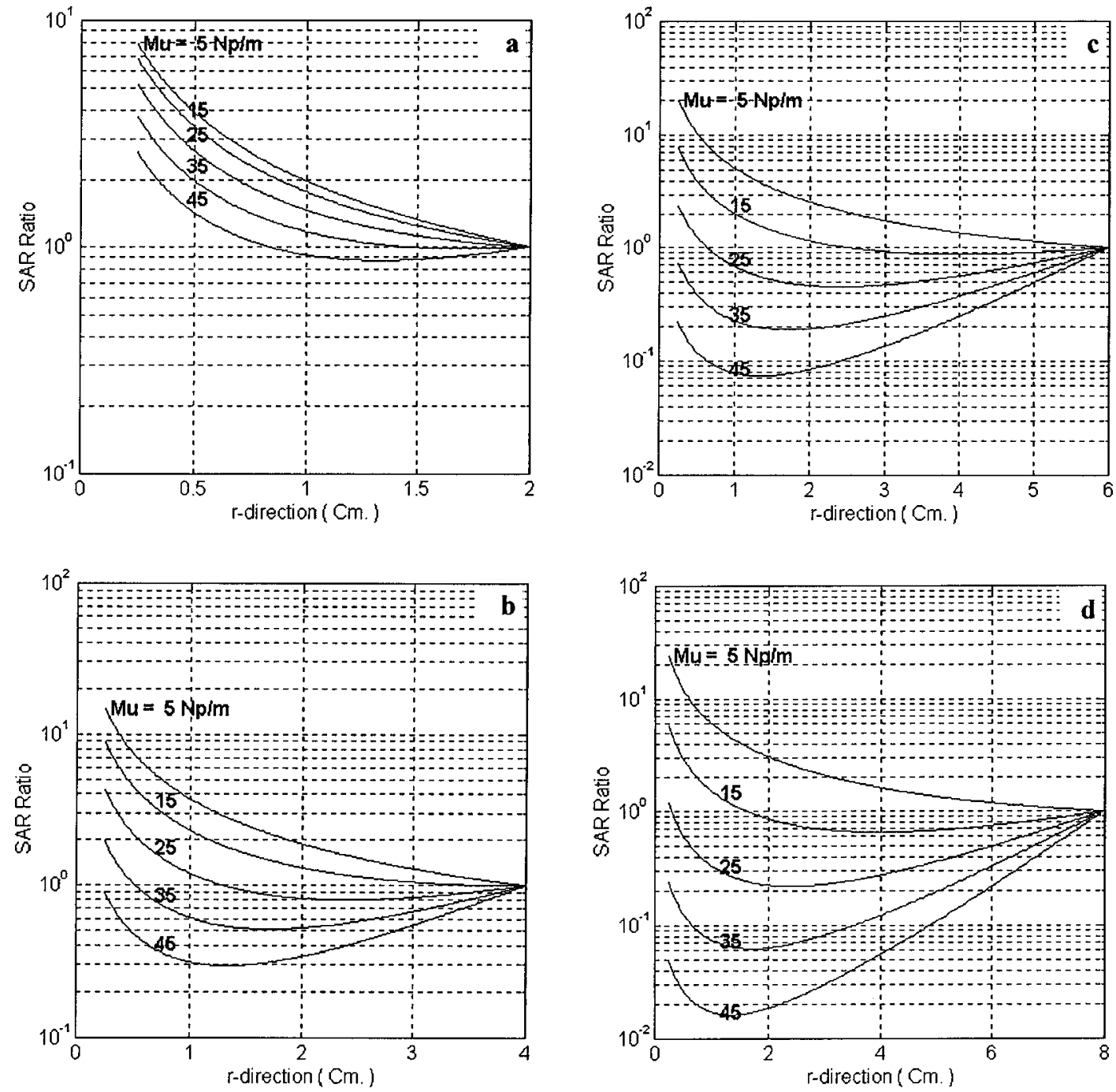

FIG. 2. The distribution of the SAR ratio for the shifting distance $(d)$ equal to zero, the active portion of transducer $360^{\circ}$, and the attenuation of ultrasound in breast tissue is varied from $5,15,25,35$, to $45 \mathrm{~Np} / \mathrm{m}$, while the diameter of breast is $4,8,12$, and $16 \mathrm{~cm}$, respectively.

ducer's active portion, $180^{\circ}-\varphi / 2 \leqslant \theta \leqslant 180^{\circ}+\varphi / 2$ :

$$
\begin{aligned}
& \mathrm{SR}\left(\mu, R, d, \varphi ; r, \theta ; R-d, 0^{\circ}\right) \\
& \quad=\frac{I(\mu, R, d, \varphi ; r, \theta)}{I\left(\mu, R, d, \varphi ; R-d, 0^{\circ}\right)} \\
& =\mathrm{GG}\left(r, \theta ; R-d, 0^{\circ}\right) \cdot e^{-2 \mu\left(S^{\prime}+r\right),} \\
& \mathrm{GG}\left(r, \theta ; R-d, 0^{\circ}\right)=\frac{R-d}{r} .
\end{aligned}
$$

Equations (3a), (6a), (8a), and (8c) investigate the relationship between the parameters and the distribution of the SAR ratio, as well as the treatable domain for a given set of control parameters.

\section{RESULTS AND DISCUSSION}

\section{A. One-dimensional power deposition}

To examine the effects of breast size and ultrasound frequency on the distribution of the SAR ratio within the breast

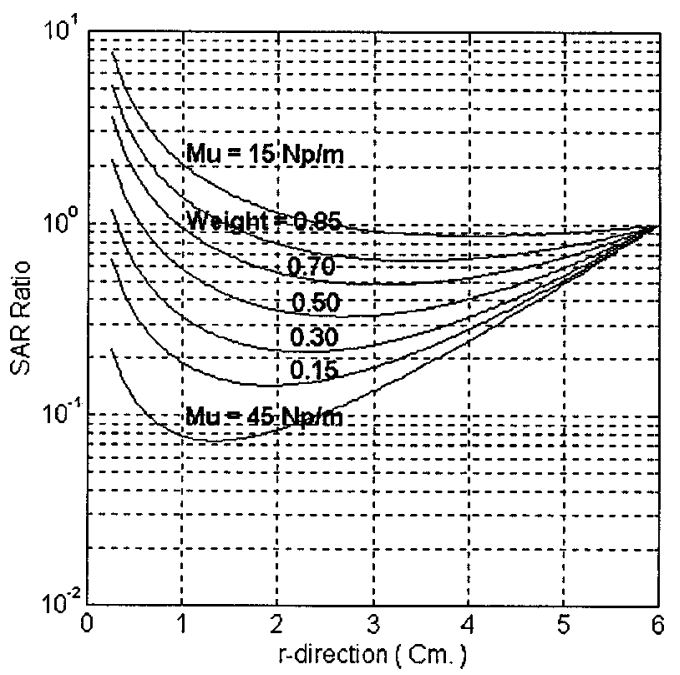

FIG. 3. The distributions of the SAR ratio for a cylindrical ultrasound transducer operating at the fundamental (attenuation $15 \mathrm{~Np} / \mathrm{m}$ ) and harmonic resonance frequency (attenuation $45 \mathrm{~Np} / \mathrm{m}$ ) on a power weighting basis. 

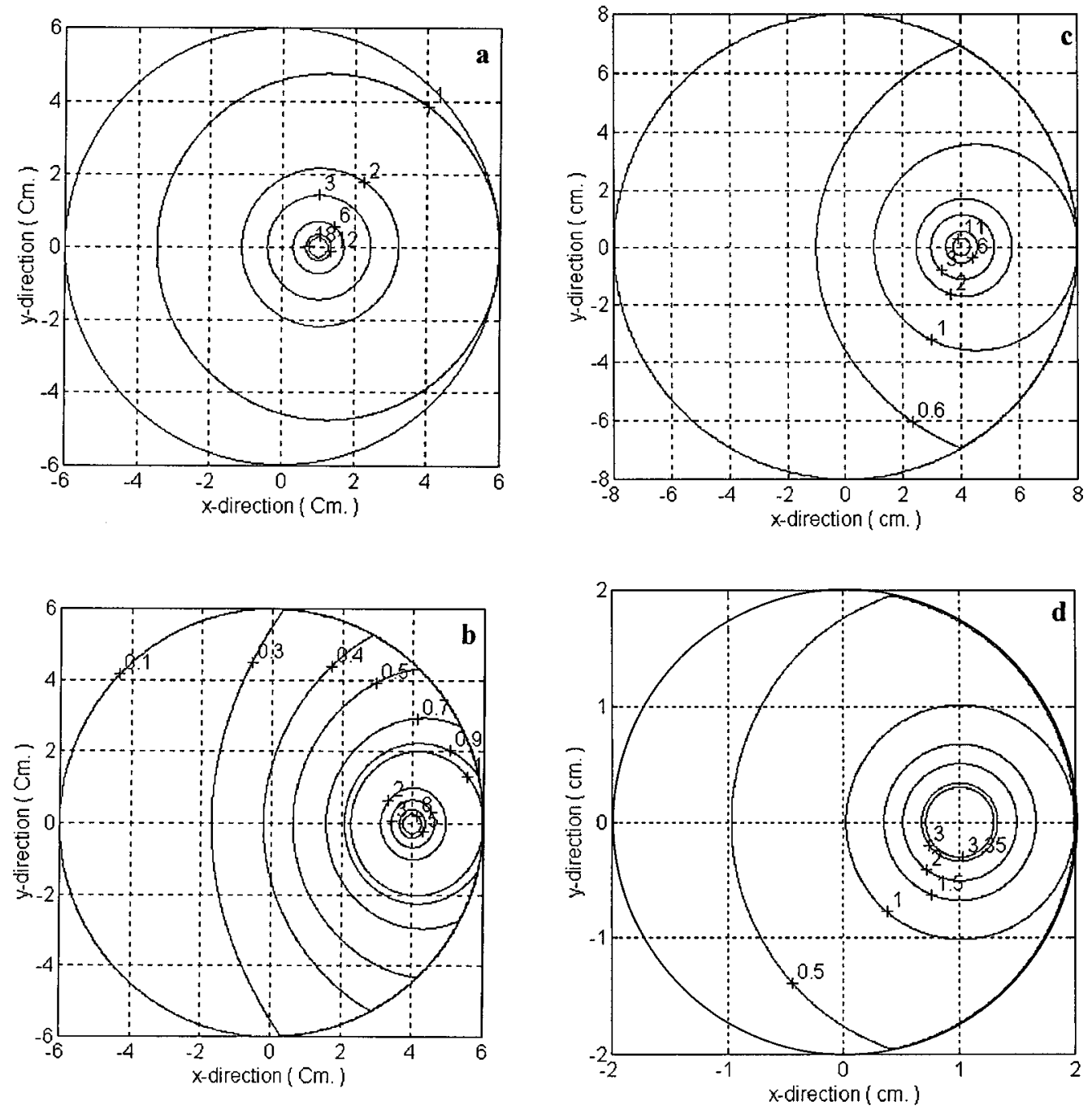

FIG. 4. The distribution of the SAR ratio for the attenuation of ultrasound in breast tissue $5 \mathrm{~Np} / \mathrm{m}$ and the active portion of transducer $360^{\circ}$; (a) and (b) the breast's diameter equal to $12 \mathrm{~cm}$, while the shifting distance is 1.0 and $4.0 \mathrm{~cm}$, respectively; (c) a large breast (diameter $16 \mathrm{~cm}$ ) with a shifting distance 4.0 $\mathrm{cm}$, and (d) a small breast (diameter $4 \mathrm{~cm}$ ) with a shifting distance $1.0 \mathrm{~cm}$.

when a cylindrical ultrasound transducer is used, the breast's diameter $(2 R)$ is varied from $4,8,12$, to $16 \mathrm{~cm}$, and the ultrasound attenuation $(\mu)$ is from $5,15,25,35$, to $45 \mathrm{~Np} / \mathrm{m}$. (With regards to the attenuation of breast tissue at $1 \mathrm{MHz}$ approximately $10 \mathrm{~Np} / \mathrm{m}$ and at $2 \mathrm{MHz}$ approximately 20 $\mathrm{Np} / \mathrm{m},{ }^{12}$ this requires an approximate frequency range of $0.5-4.5 \mathrm{MHz}$ ). The central axis of cylindrical transducer coincides with that of breast (the shifting distance $d=0$ ), and hence the ultrasound power deposition within the breast is one dimensional (symmetrical with the central axis). Figures 2(a)-2(d) are the distributions of the SAR ratio based on Eq. (3a). Figures 2(a)-2(d) reveal that the SAR ratio for low attenuations is always higher than that for high attenuations for all different breast sizes, and show that the ratio increases from the surface to the central portion of the breast for low attenuation cases but, for high attenuation cases, it decreases initially and then increases and the value is lower than 1.0. In Fig. 2(a) the SAR ratio for the small breast case (diameter 4 $\mathrm{cm}$ ) increases and is always higher than 1.0 for all attenuations except $45 \mathrm{~Np} / \mathrm{m}$. In Fig. 2(d) the ratio for the large breast case (diameter $16 \mathrm{~cm}$ ) decreases initially and then increases for all attenuations except $5 \mathrm{~Np} / \mathrm{m}$. For low attenuations, a single peak of the SAR ratio appears in the central portion of the breast for all different breast sizes. However, two peaks appear for large breasts with high attenuations: one higher peak appears on the breast surface and the other in the central portion of the breast.

Figure 2 depicts the relationship between treatable domain and frequency for different breast sizes if an appropriate definition is given for the treatable region. For instance, we might define the domain with the SAR ratio higher than 3.0 as the treatable region. The criterion would indicate that (a) lower frequencies should be used in the treatment of the central portion of the breast for all different breast sizes, and (b) the treatable region size is dependent on the frequency and a lower frequency owns a larger region. For large breasts with high frequencies, the SAR ratio is always smaller than 1.0 , we might define the portion with a SAR ratio higher than 0.3 as the treatable region. The criterion would give that (a) higher frequencies can be used in the treatment of the super- 

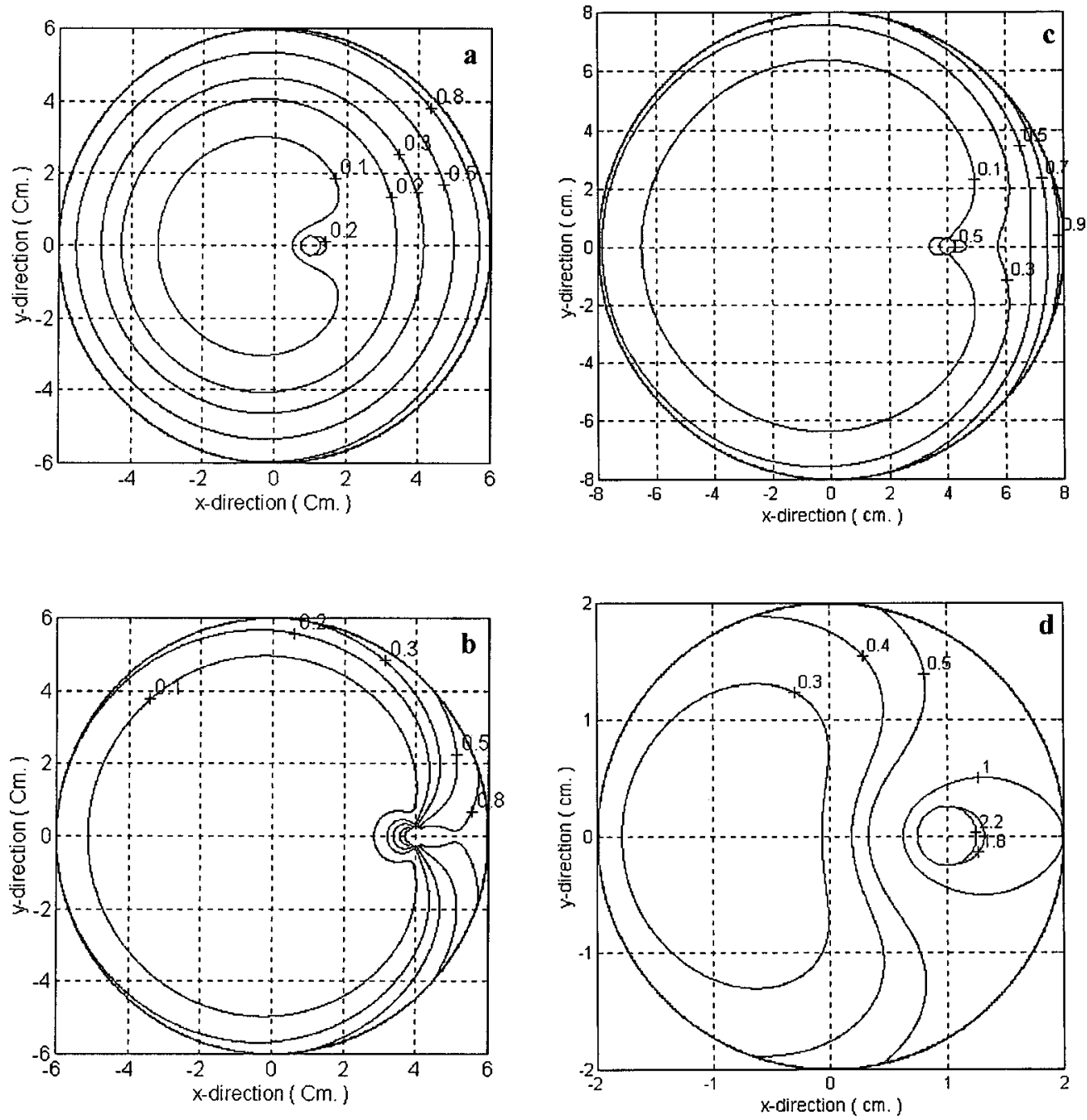

FIG. 5. The distribution of the SAR ratio for the attenuation of ultrasound in breast tissue $45 \mathrm{~Np} / \mathrm{m}$ and the active portion of transducer $360^{\circ}$; (a) and (b) the breast's diameter equal to $12 \mathrm{~cm}$, while the shifting distance is 1.0 and $4.0 \mathrm{~cm}$, respectively; (c) a large breast (diameter $16 \mathrm{~cm}$ ) with a shifting distance 4.0 $\mathrm{cm}$; and $(\mathrm{d})$ a small breast (diameter $4 \mathrm{~cm}$ ) with a shifting distance $1.0 \mathrm{~cm}$.

ficial portion of large breasts, (b) a lower frequency owns a larger treatable region but the other peak of the SAR ratio in the central portion of the breast might be too high, and (c) high frequencies cannot be used in the treatment of the superficial portion for small breasts. These figures display the treatable region for a given breast size and the corresponding attenuation, which is called the optimal attenuation, indicating the optimal driving frequency to yield the given treatable region. Hence, the optimal driving frequency is the one to be used to obtain a given treatment region for a given breast size. The above finding suggests that the treatable portion of the central or superficial breast can be determined by selecting an appropriate frequency while the breast size is known.

Figure 3 is the distributions of the SAR ratio for a cylindrical transducer operating at the fundamental and harmonic resonance frequencies, attenuation 15 and $45 \mathrm{~Np} / \mathrm{m}$, respectively. SAR ratios for different power weightings are also shown. A power weighting close to $15 \mathrm{~Np} / \mathrm{m}$ results in a deep heating, and the other way close to $45 \mathrm{~Np} / \mathrm{m}$ will obtain a superficial heating. The results demonstrate that the treat- able region can be controlled by using a single cylindrical ultrasound transducer operating in some combination of fundamental and harmonic resonance frequency on a power weighting basis. ${ }^{13}$

\section{B. Two-dimensional power deposition}

As the central axis of the cylindrical transducer is shifted a distance of $d$ meters from that of breast, the ultrasound power deposition within the breast becomes two dimensional. Frequency [or attenuation $(\mu)$ ], shifting distance $(d)$, and the breast's diameter $(2 R)$ are the factors potentially influencing the distribution of the SAR ratio. Figures 4(a) and 4(b) based on Eq. (6a) for a breast's diameter of $12 \mathrm{~cm}$ and attenuation $5 \mathrm{~Np} / \mathrm{m}$ and shifting distances 1.0 and 4.0 $\mathrm{cm}$, provide the simulation results revealing that the distribution of the SAR ratio is dependent on the shifting distance. Figures 4(c) and 4(d) are the distributions of the SAR ratio for a large breast (diameter $16 \mathrm{~cm}$ ) with a shifting distance $4.0 \mathrm{~cm}$ and a small breast (diameter $4 \mathrm{~cm}$ ) with a shifting 

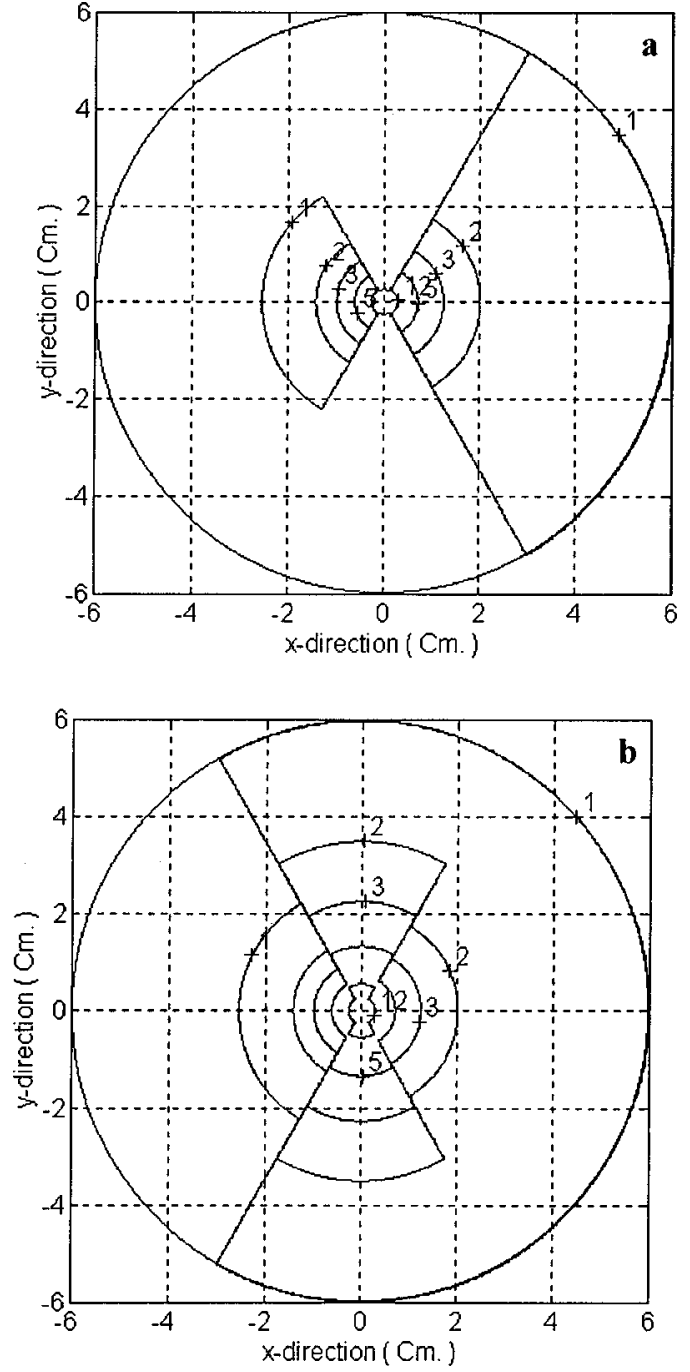

FIG. 6. The distribution of the SAR ratio for the shifting distance equal to $0.0 \mathrm{~cm}$, the breast's diameter $12 \mathrm{~cm}$, and the attenuation of ultrasound 5 $\mathrm{Np} / \mathrm{m}$, while the active portion of the transducer is $120^{\circ}$ and $240^{\circ}$, respectively.

distance of $1.0 \mathrm{~cm}$, respectively. Figures 4(a)-4(d) display that (a) the pattern of the SAR ratio is shifted with the transducer, (b) both the region of the SAR ratio higher than 1.0 and the peak value of the SAR ratio become smaller as the shifting distance increases, and (c) the region of the high SAR ratio is shifted with the transducer for all different breast sizes.

Figures 5(a) and 5(b), based on Eq. (6a) for an attenuation of $45 \mathrm{~Np} / \mathrm{m}$ with a breast diameter of $12 \mathrm{~cm}$ and shifting distances 1.0 and $4.0 \mathrm{~cm}$, reveal that the distribution of the SAR ratio is dramatically changed with the shifting distance. The central portion of the SAR ratio is shifted with the cylindrical transducer and its value increases with the shifting distance, while the value on the breast surface decreases. The region of the SAR ratio higher than 0.3 converges from the superficial region of an entire breast to a small region in the right breast. Figures 5(c) and 5(d) are the distributions of the SAR ratio for a large breast (diameter $16 \mathrm{~cm}$ ) with a shifting distance $4.0 \mathrm{~cm}$ and a small breast (diameter $4 \mathrm{~cm}$ ) with a
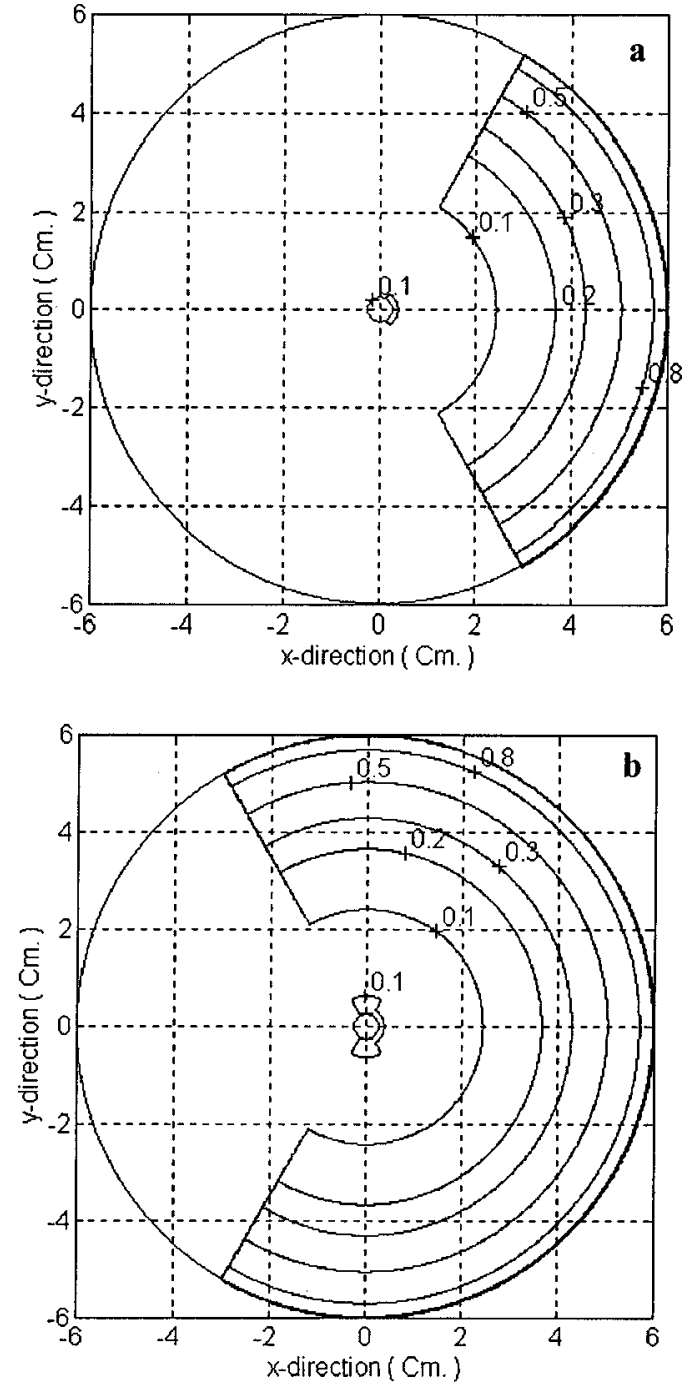

FIG. 7. The distribution of the SAR ratio for the shifting distance equal to $0.0 \mathrm{~cm}$, the breast's diameter $12 \mathrm{~cm}$, and the attenuation of ultrasound 45 $\mathrm{Np} / \mathrm{m}$, while the active portion of the transducer is $120^{\circ}$ and $240^{\circ}$, respectively.

shifting distance $1.0 \mathrm{~cm}$, respectively. The region of the SAR ratio higher than 0.3 is located on the superficial portion of the breast and shifted with the transducer for the large breast case. However, a region with the SAR ratio higher than 1.0, which is shifted with the transducer, appears for the small breast case. This indicates that a planar transducer is more appropriate for small breasts to obtain a superficial region with a high SAR ratio.

In addition to the frequency and the shifting distance of the transducer, the active portion $(\varphi)$ of the cylindrical transducer can also be employed to control the distribution of the SAR ratio. To examine the effects of the active portion of the transducer on the distribution of the SAR ratio, the active portion $(\varphi)$ is varied from $120^{\circ}$ to $240^{\circ}$ for a breast diameter $12.0 \mathrm{~cm}$ with a shifting distance $0.0 \mathrm{~cm}$ and attenuations 5 and $45 \mathrm{~Np} / \mathrm{m}$. Figures 6(a) and 6(b), obtained from Eqs. (8a) and $(8 \mathrm{c})$ for attenuation $5 \mathrm{~Np} / \mathrm{m}$, indicate that the domain with a SAR ratio higher than 3.0 is located in the central portion of the breast and its size is proportional to the active 
portion $(\varphi)$ of transducer. Figure 6(a) reveals that the right portion of the breast facing the active portion of the transducer owns a higher SAR ratio. Figure 6(b), with an active portion $240^{\circ}$, displays three distinct regions: facing on, backing to the active portion of transducer, and a combination of both. The combination region owns the highest SAR ratio. Figures $7(\mathrm{a})$ and $7(\mathrm{~b})$, for attenuation of $45 \mathrm{~Np} / \mathrm{m}$, indicate that the domain with the SAR ratio exceeding 0.3 is always located in the superficial region of the breast and its size is proportional to the active portion $(\varphi)$ of transducer.

The above study demonstrates that: (1) the driving frequency, the shifting distance, and the active portion of the cylindrical transducer are the potential control parameters for the distribution of the SAR ratio; (2) for superficial heating treatments, high frequencies should be used and the active portion $(\varphi)$ of the transducer can be changed to obtain a region with an appropriate SAR ratio to cover the treatment region; (3) for deep heating treatments, low frequencies should be used and the shifting of transducer can move the region of high SAR ratio and its pattern can also be controlled by changing the active portion of transducer; (4) the distribution of the SAR ratio is also dependent on the breast size; (5) for a small breast, a high SAR ratio on the superficial region of breast cannot be formed using a cylindrical transducer even though high frequencies are used. In this case, a planar transducer with high frequencies is suggested.

The study of SAR ratio distribution is based on an ideal ultrasound power deposition from a cylindrical transducer. The interference maxima and minima in the near field for the cylindrical transducer oscillate within an envelope function ${ }^{14,15}$ and will be further studied using the wave equation while appropriate lengths of the sides of curved rectangular transducer elements are employed to form the entire cylindrical ultrasound transducer.

\section{CONCLUSION}

The above SAR ratio study for a cylindrical transducer employs a relatively simple model of power deposition to investigate the optimal driving frequency for the ultrasound transducer, as well as the treatable domain of breast with respect to the breast size, the shifting distance, and the active portion of transducer. Simulation results demonstrate the optimal attenuation to select the appropriate driving frequency for a given SAR ratio pattern. The above results also indicate the treatable domain for a given driving frequency, shifting distance, and active portion of transducer. Findings of this study can be used as a guideline (1) to know whether or not the desired treatment region is treatable, (2) to select the optimal driving frequency for the transducer and an appropriate shifting and active portion of transducer for a treatment while the desired treatment size and depth are known during the clinical treatment, (3) to specifically design an appropriate ultrasound heating system for breast tumor treat- ments. These SAR ratio patterns are the initial results that could then be modified for additional modeling that would include temperature calculations, the arrangement of transducer elements, amplitude control of each element, etc.

\section{ACKNOWLEDGMENTS}

The authors would like to thank the National Science Council and the Department of Health of the Republic of China for partially supporting this research under Contract Nos. NSC 85-2213-E-002-033 and DOH 85-HR-310, respectively.

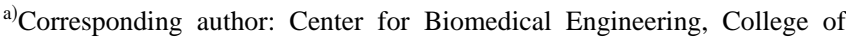
Medicine, National Taiwan University, No. 1, Sec. 1, Jan-Ai Road, Taipei, Taiwan.

${ }^{1}$ M. Mitsumori, M. Hiraoka, Y. Okuno, Y. Nishimura, Y. P. Li, S. Fujishiro, Y. Nagata, and M. Abe, "A phase I and II clinical trial of a newly developed ultrasound hyperthermia system with an improved planar transducer," Proceedings of the Seventh International Congress on Hyperthermic Oncology, Rome, Italy, 1996 (unpublished), Vol. 2, pp. 396-398.

${ }^{2}$ X.-Q. Lu, E. C. Burdette, B. A. Bornstein, J. L. Hansen, and G. K. Svensson, "Design of an ultrasonic therapy system for breast cancer treatment," Int. J. Hyperthermia 12, 375-399 (1996).

${ }^{3}$ J. L. Hansen, E. C. Burdette, P. Neubauer, X.-Q. Lu, B, A. Bornstein, and G. K. Svensson, "Experimental verification of a cylindrical multitransducer ultrasound breast hyperthermia treatment," in Ref. 1, pp. 388390.

${ }^{4}$ P. P. Lele, "Physical aspects and clinical studies with ultrasound hyperthermia," in Hyperthermia in Cancer Therapy, edited by F. C. Storm (Hall, Boston, 1983), pp. 333-367.

${ }^{5}$ P. Fessenden, E. R. Lee, T. L. Anderson, J. W. Strohbehn, J. L. Meyer, T. V. Samulski, and J. B. Marmor, "Experience with multitransducer ultrasound system for localized hyperthermia of deep tissues," IEEE Trans. Biomed. Eng. 31, 126-134 (1984).

${ }^{6}$ K. H. Hynynen, R. B. Roemer, D. Anhalt, C. Johnson, Z. X. Xu, W. Swindell, and T. Cetas, "A scanned focused multiple transducer ultrasonic system for localized hyperthermia treatments,' Int. J. Hyperthermia 3, 21-35 (1987).

${ }^{7}$ A. W. Dutton, R. B. Roemer, and F. A. Gibbs, "A clinically integrated scanned focused ultrasound hyperthermia," in Ref. 1, Vol. 1, p. 92.

${ }^{8}$ P. M. Corry, B. Barlogie, E. J. Tilchen, and E. P. Armour, "Ultrasound induced hyperthermia for the treatment of human superficial tumors," Int. J. Radiat. Oncol., Biol., Phys. 8, 1225-1229 (1982).

${ }^{9}$ P. Munro, R. P. Hill, and J. W. Hunt, "The development of improved ultrasound heaters suitable for superficial tissue heating," Med. Phys. 9, 888-897 (1982).

${ }^{10}$ H. R. Underwood, E. C. Burdette, K. B. Ocheltree, and R. L. Magin, “A multielement ultrasonic hyperthermia applicator with independent element control,"' Int. J. Hyperthermia 3, 257-267 (1987).

${ }^{11} \mathrm{~K}$. H. Hynynen, "Biophysics and technology of ultrasound hyperthermia," in Method of External Hyperthermic Heating, edited by M. Gautherie (Springer, Berlin, 1990), pp. 61-116.

${ }^{12}$ F. S. Foster, and J. W. Hunt, "Transmission of ultrasound beams through human tissue-focusing and attenuation studies," Ultrasound Med. Biol. 5, 257-268 (1979).

${ }^{13}$ T. Sano, N. Takayama, and M. Saito, "Depth control of heating with a non-focused ultrasound transducer operating at fundamental and harmonic resonance frequencies,"' in Ref. 1, pp. 379-381.

${ }^{14}$ L. E. Kinsler and A. R. Frey, Fundamentals of Acoustics (Wiley, New York, 1982), pp. 92-117.

${ }^{15}$ T. F. Hueter and R. H. Bolt, Sonics: Techniques for the Use of Sound and Ultrasound in Engineering and Science (Wiley, New York, 1955), pp. 263-274. 